Supplement of Clim. Past, 13, 1213-1226, 2017

https://doi.org/10.5194/cp-13-1213-2017-supplement

(C) Author(s) 2017. This work is distributed under

the Creative Commons Attribution 3.0 License.

(c) (1)

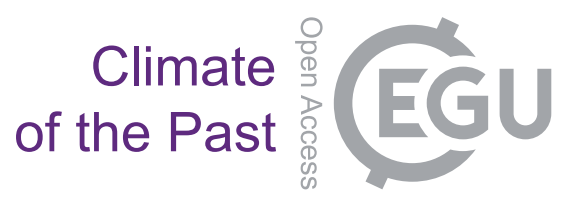

Supplement of

\title{
Sources and characteristics of terrestrial carbon in Holocene-scale sediments of the East Siberian Sea
}

\author{
Kirsi Keskitalo et al. \\ Correspondence to: Örjan Gustafsson (orjan.gustafsson@aces.su.se)
}

The copyright of individual parts of the supplement might differ from the CC BY 3.0 License. 
Supplementary information

Tables and figures

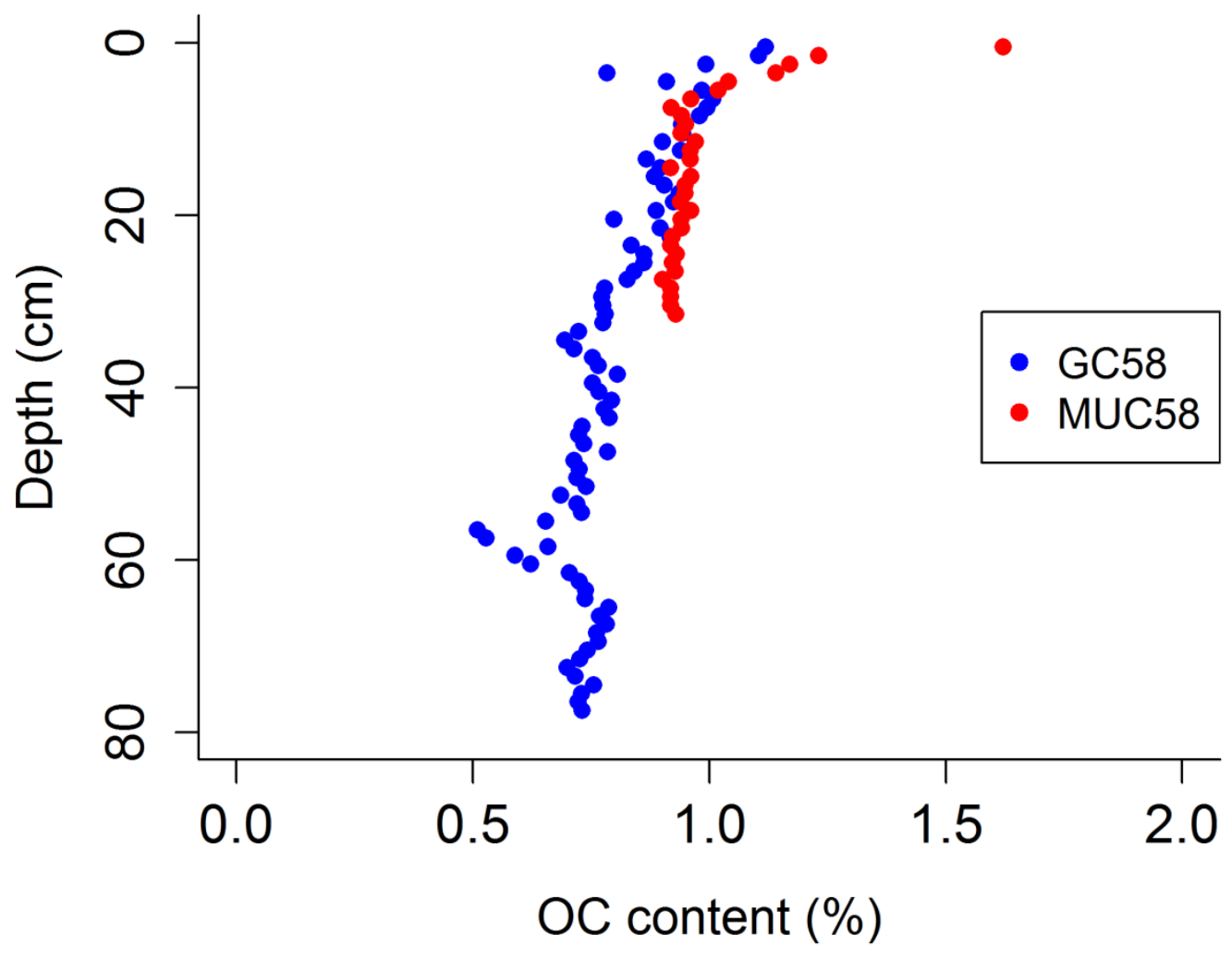

Figure S1. Comparison between the organic carbon (OC) content (\%) of the sediment cores GC58 and MUC58. Based on the comparison, we deduced that the top $3 \mathrm{~cm}$ of GC58 were lost during sampling. 
Table S1. Total organic carbon (TOC) content, stable carbon isotopes $\left(\delta^{13} \mathrm{C}\right)$ and calibrated radiocarbon isotopes of bulk carbon (cal $\Delta^{14} \mathrm{C}$ ) in the sediment core GC58 that were used for source apportionment calculations.

\begin{tabular}{|c|c|c|c|}
\hline $\begin{array}{c}\text { Corrected } \\
\text { depth* }(\mathrm{cm})\end{array}$ & TOC $(\%)$ & $\delta^{13} \mathrm{C}(\%)$ & $\begin{array}{c}\text { Cal } \Delta^{14} \mathrm{C} \\
(\%)\end{array}$ \\
\hline 3.5 & 1.12 & -23.23 & -400.17 \\
\hline 4.5 & 1.10 & -23.07 & \\
\hline 5.5 & 0.99 & -23.25 & \\
\hline 6.5 & 0.78 & -23.10 & \\
\hline 7.5 & 0.91 & -23.16 & \\
\hline 8.5 & 0.99 & -23.32 & \\
\hline 9.5 & 1.01 & -23.12 & \\
\hline 10.5 & 1.00 & -23.42 & \\
\hline 11.5 & 0.98 & -23.48 & \\
\hline 12.5 & 0.94 & -23.39 & \\
\hline 13.5 & 0.94 & -23.19 & -568.02 \\
\hline 14.5 & 0.90 & -23.32 & \\
\hline 15.5 & 0.94 & -23.38 & \\
\hline 16.5 & 0.87 & -23.49 & \\
\hline 17.5 & 0.90 & -23.61 & \\
\hline 18.5 & 0.88 & -23.56 & \\
\hline 19.5 & 0.91 & -23.61 & \\
\hline 20.5 & 0.94 & -23.44 & \\
\hline 21.5 & 0.93 & -23.62 & \\
\hline 22.5 & 0.89 & -23.57 & \\
\hline 23.5 & 0.80 & -23.50 & -604.39 \\
\hline 24.5 & 0.90 & -23.56 & \\
\hline 25.5 & 0.92 & -23.59 & \\
\hline 26.5 & 0.84 & -23.76 & \\
\hline 27.5 & 0.86 & -23.57 & \\
\hline 28.5 & 0.86 & -23.88 & \\
\hline 29.5 & 0.84 & -23.61 & \\
\hline 30.5 & 0.83 & -24.33 & \\
\hline 31.5 & 0.78 & -24.08 & \\
\hline 32.5 & 0.77 & -24.22 & \\
\hline 33.5 & 0.78 & -23.89 & -679.07 \\
\hline 34.5 & 0.78 & -24.14 & \\
\hline 35.5 & 0.78 & -24.24 & \\
\hline 36.5 & 0.72 & -24.73 & \\
\hline 37.5 & 0.70 & -24.54 & \\
\hline 38.5 & 0.71 & -24.63 & -747.85 \\
\hline 39.5 & 0.75 & -24.37 & \\
\hline 40.5 & 0.77 & -24.82 & \\
\hline 41.5 & 0.81 & -24.75 & \\
\hline 42.5 & 0.75 & -24.95 & \\
\hline 43.5 & 0.77 & -25.07 & -586.93 \\
\hline 44.5 & 0.79 & -24.91 & \\
\hline
\end{tabular}




\begin{tabular}{|c|c|c|c|}
\hline 45.5 & 0.78 & -24.91 & \\
\hline 46.5 & 0.79 & -24.93 & \\
\hline 47.5 & 0.73 & -24.86 & -607.58 \\
\hline 48.5 & 0.72 & -24.93 & \\
\hline 49.5 & 0.74 & -25.01 & \\
\hline 50.5 & 0.79 & -24.85 & \\
\hline 51.5 & 0.71 & -25.08 & \\
\hline 52.5 & 0.73 & -25.05 & \\
\hline 53.5 & 0.72 & -25.11 & -680.66 \\
\hline 54.5 & 0.74 & -24.85 & \\
\hline 55.5 & 0.69 & -25.34 & \\
\hline 56.5 & 0.72 & -25.44 & \\
\hline 57.5 & 0.73 & -25.51 & \\
\hline 58.5 & 0.65 & -25.66 & \\
\hline 59.5 & 0.51 & -25.65 & \\
\hline 60.5 & 0.53 & -25.68 & \\
\hline 61.5 & 0.66 & -25.55 & \\
\hline 62.5 & 0.59 & -25.65 & \\
\hline 63.5 & 0.62 & -25.74 & -771.72 \\
\hline 64.5 & 0.71 & -25.59 & \\
\hline 65.5 & 0.73 & -25.68 & \\
\hline 66.5 & 0.74 & -25.66 & \\
\hline 67.5 & 0.74 & -25.66 & \\
\hline 68.5 & 0.79 & -25.53 & \\
\hline 69.5 & 0.77 & -25.51 & \\
\hline 70.5 & 0.78 & -25.63 & \\
\hline 71.5 & 0.76 & -25.60 & \\
\hline 72.5 & 0.77 & -25.59 & \\
\hline 73.5 & 0.74 & -25.66 & -764.43 \\
\hline 74.5 & 0.73 & -25.60 & \\
\hline 75.5 & 0.70 & -25.59 & \\
\hline 76.5 & 0.72 & -25.70 & \\
\hline 77.5 & 0.76 & -25.65 & \\
\hline 78.5 & 0.73 & -25.84 & \\
\hline 79.5 & 0.72 & -25.75 & \\
\hline 80.5 & 0.73 & -25.58 & \\
\hline
\end{tabular}

*Corrected depth is the original depth $+3 \mathrm{~cm}$ to account for core top loss during sampling (Sect. 2.4). 
Table S2. The amount of excess ${ }^{210} \mathrm{~Pb}\left(\mathrm{~Bq} \mathrm{~g}^{-1}\right)$ and natural logarithm (ln) of excess ${ }^{210} \mathrm{~Pb}$ in the sediment core MUC58 and age chronology (CRC and CIC models) presented as age (yr) and resulting year of deposition. The CRC model assumes a constant rate of supply of ${ }^{210} \mathrm{~Pb}$ fallout, as the CIC assumes the initial concentration of ${ }^{210} \mathrm{~Pb}$ to be constant (Appleby \& Oldfield, 1978). The highest ${ }^{137} \mathrm{Cs}$ peak was in 4.5 cm depth.

\begin{tabular}{cccccccc}
\hline $\begin{array}{c}\text { Depth } \\
(\mathbf{c m})\end{array}$ & $\begin{array}{c}\text { Excess }{ }^{\mathbf{2 1 0}} \mathbf{P b} \\
\left(\mathbf{B q} \mathbf{~ g}^{\mathbf{- 1}}\right)\end{array}$ & $\begin{array}{c}\text { Ln of } \\
\text { excess }{ }^{\mathbf{2 1 0}} \mathbf{P b}\end{array}$ & $\begin{array}{c}\text { Age (yr) } \\
\text { with CRC } \\
\text { model }\end{array}$ & $\begin{array}{c}\text { Resulting } \\
\text { year with } \\
\text { CRC model }\end{array}$ & $\begin{array}{c}\text { Age (yr) } \\
\text { with CIC } \\
\text { model }\end{array}$ & $\begin{array}{c}\text { Resulting } \\
\text { year with } \\
\text { CIC model }\end{array}$ & $\begin{array}{c}\text { OC flux } \\
\left(\mathbf{g ~ m}^{-2} \mathbf{y r}^{-1}\right)\end{array}$ \\
\hline 0.5 & 0.062 & -2.78 & 0 & 2014 & 4 & 2010 & 6.1 \\
1.5 & 0.061 & -2.79 & 6 & 2008 & 12 & 2002 & 4.6 \\
2.5 & 0.054 & -2.92 & 14 & 2000 & 20 & 1994 & 4.4 \\
3.5 & 0.049 & -3.02 & 23 & 1991 & 28 & 1986 & 4.3 \\
4.5 & 0.024 & -3.73 & 35 & 1979 & 36 & 1978 & 3.9 \\
5.5 & 0.016 & -4.11 & 42 & 1972 & 44 & 1970 & 3.8 \\
6.5 & 0.014 & -4.24 & 49 & 1965 & 52 & 1962 & 3.6 \\
7.5 & 0.021 & -3.86 & 55 & 1959 & 60 & 1954 & 3.4 \\
8.5 & 0.016 & -4.145 & 69 & 1945 & 68 & 1946 & 3.5 \\
9.5 & 0.006 & -5.16 & 85 & 1929 & 75 & 1939 & 3.6 \\
10.5 & 0.001 & -6.67 & 94 & 1920 & 83 & 1931 & 3.5 \\
11.5 & 0.012 & -4.41 & 96 & 1918 & 91 & 1923 & 3.6 \\
12.5 & 0.005 & -5.25 & 134 & 1880 & 99 & 1915 & 0.4 \\
\hline
\end{tabular}


Table S3. Biomarker data for the sediment core GC58. $\mathrm{S}=$ syringyl phenols, $\mathrm{V}=$ vanillyl phenols, $\mathrm{C}=$ cinnamyl phenols, 3,5-Bd = 3,5-Dihydroxybenzoic acid, $\mathrm{Cut}=$ sum of all cutin acids, $\mathrm{FA}=$ sum of all CuO-oxidation-derived fatty acids, $\mathrm{Lig}=$ sum of all lignin phenols, $\mathrm{Sd}=$ syringic acid, $\mathrm{Sl}=$ syringaldehyde, $\mathrm{Vd}=$ vanillic acid, Vl = vanillin (see Supplementary Table S4 for full list of compounds and their origin).

\begin{tabular}{|c|c|c|c|c|c|c|c|c|c|c|c|c|}
\hline $\begin{array}{c}\text { Corrected } \\
\text { depth* } \\
\mathrm{cm} \\
\end{array}$ & $\begin{array}{c}\mathbf{S} \\
\mathrm{mg} \mathrm{g} \mathrm{OC}^{-1} \\
\end{array}$ & $\begin{array}{c}\mathbf{V} \\
\mathrm{mg} \mathrm{g} \mathrm{OC}^{-1} \\
\end{array}$ & $\begin{array}{c}\mathbf{C} \\
\operatorname{mg~g~OC~}^{-1} \\
\end{array}$ & $\begin{array}{c}\mathbf{3 , 5}-\mathbf{B d} \\
\mathrm{mg} \mathrm{g} \mathrm{OC}^{-1} \\
\end{array}$ & $\begin{array}{c}\text { Cut } \\
\mathrm{mg} \mathrm{g} \mathrm{OC}^{-1}\end{array}$ & $\begin{array}{c}\text { FA } \\
\mathrm{mg} \mathrm{g} \mathrm{OC}^{-1}\end{array}$ & $\begin{array}{c}\text { Lig } \\
\operatorname{mg~g~OC~}^{-1} \\
\end{array}$ & $\mathbf{S} / \mathbf{V}$ & $\mathrm{C} / \mathrm{V}$ & $\mathbf{S d} / \mathbf{S l}$ & $\mathbf{V d} / \mathbf{V l}$ & 3,5-Bd/V \\
\hline 3.5 & 0.05 & 0.13 & 0.04 & 0.1225 & 0.27 & 5.35 & 0.2244 & 0.42 & 0.31 & 0.59 & 0.95 & 0.94 \\
\hline 4.5 & 0.08 & 0.12 & 0.07 & 0.0898 & 0.30 & 3.17 & 0.2700 & 0.66 & 0.60 & 0.64 & 0.95 & 0.75 \\
\hline 5.5 & 0.07 & 0.08 & 0.03 & 0.1148 & 0.24 & 2.55 & 0.1770 & 0.90 & 0.37 & 0.59 & 0.00 & 1.47 \\
\hline 6.5 & 0.07 & 0.12 & 0.03 & 0.1008 & 0.30 & 2.55 & 0.2318 & 0.59 & 0.26 & 0.63 & 0.78 & 0.81 \\
\hline 7.5 & 0.07 & 0.12 & 0.03 & 0.1486 & 0.30 & 2.06 & 0.2222 & 0.59 & 0.23 & 0.52 & 0.88 & 1.22 \\
\hline 8.5 & 0.05 & 0.12 & 0.03 & 0.1055 & 0.27 & 1.38 & 0.2017 & 0.39 & 0.24 & 0.66 & 1.55 & 0.85 \\
\hline 9.5 & 0.04 & 0.13 & 0.04 & 0.0696 & 0.27 & 1.26 & 0.2071 & 0.28 & 0.28 & 0.69 & 2.10 & 0.52 \\
\hline 10.5 & 0.05 & 0.14 & 0.04 & 0.1043 & 0.28 & 1.41 & 0.2349 & 0.37 & 0.31 & 0.74 & 1.66 & 0.75 \\
\hline 11.5 & 0.10 & 0.21 & 0.09 & 0.1083 & 0.34 & 1.31 & 0.4051 & 0.49 & 0.43 & 0.59 & 1.12 & 0.51 \\
\hline 12.5 & 0.05 & 0.15 & 0.03 & 0.0978 & 0.34 & 1.53 & 0.2304 & 0.33 & 0.22 & 0.75 & 1.17 & 0.66 \\
\hline 13.5 & 0.04 & 0.09 & 0.03 & 0.0866 & 0.29 & 1.35 & 0.1603 & 0.43 & 0.32 & 0.64 & 1.92 & 0.95 \\
\hline 14.5 & 0.05 & 0.14 & 0.05 & 0.1276 & 0.35 & 1.43 & 0.2386 & 0.39 & 0.33 & 0.75 & 1.30 & 0.92 \\
\hline 15.5 & 0.05 & 0.10 & 0.03 & 0.0917 & 0.27 & 1.12 & 0.1763 & 0.48 & 0.35 & 0.66 & 1.35 & 0.95 \\
\hline 16.5 & 0.05 & 0.16 & 0.03 & 0.1005 & 0.28 & 1.40 & 0.2383 & 0.33 & 0.19 & 0.60 & 1.32 & 0.64 \\
\hline 17.5 & 0.05 & 0.14 & 0.05 & 0.1092 & 0.35 & 1.43 & 0.2356 & 0.39 & 0.34 & 0.78 & 1.59 & 0.80 \\
\hline 18.5 & 0.05 & 0.12 & 0.04 & 0.0741 & 0.33 & 1.32 & 0.2125 & 0.45 & 0.38 & 0.64 & 1.35 & 0.64 \\
\hline 19.5 & 0.05 & 0.13 & 0.04 & 0.0831 & 0.30 & 1.14 & 0.2190 & 0.38 & 0.29 & 0.71 & 1.46 & 0.63 \\
\hline 20.5 & 0.05 & 0.09 & 0.02 & 0.0969 & 0.30 & 1.20 & 0.1668 & 0.52 & 0.25 & 0.77 & 1.93 & 1.03 \\
\hline 21.5 & 0.05 & 0.10 & 0.05 & 0.0928 & 0.34 & 1.28 & 0.2033 & 0.50 & 0.46 & 0.76 & 1.78 & 0.90 \\
\hline 22.5 & 0.06 & 0.16 & 0.05 & 0.1101 & 0.37 & 1.56 & 0.2716 & 0.40 & 0.29 & 0.72 & 1.41 & 0.69 \\
\hline 23.5 & 0.07 & 0.17 & 0.05 & 0.1140 & 0.38 & 1.76 & 0.2973 & 0.41 & 0.29 & 0.76 & 1.62 & 0.65 \\
\hline 24.5 & 0.06 & 0.15 & 0.05 & 0.0931 & 0.33 & 1.19 & 0.2595 & 0.38 & 0.30 & 0.80 & 2.10 & 0.60 \\
\hline 25.5 & 0.06 & 0.15 & 0.04 & 0.0830 & 0.37 & 1.16 & 0.2581 & 0.41 & 0.26 & 0.73 & 2.45 & 0.54 \\
\hline 26.7 & 0.07 & 0.15 & 0.05 & 0.1321 & 0.41 & 1.31 & 0.2686 & 0.49 & 0.33 & 0.78 & 1.66 & 0.90 \\
\hline 27.5 & 0.06 & 0.15 & 0.05 & 0.0829 & 0.31 & 0.95 & 0.2650 & 0.41 & 0.32 & 0.79 & 1.81 & 0.54 \\
\hline
\end{tabular}




\begin{tabular}{|c|c|c|c|c|c|c|c|c|c|c|c|c|}
\hline 28.5 & 0.08 & 0.18 & 0.05 & 0.0812 & 0.16 & 1.01 & 0.3152 & 0.46 & 0.27 & 0.63 & 1.07 & 0.44 \\
\hline 29.5 & 0.08 & 0.19 & 0.06 & 0.1247 & 0.40 & 1.28 & 0.3308 & 0.41 & 0.29 & 0.85 & 1.87 & 0.64 \\
\hline 30.5 & 0.12 & 0.26 & 0.09 & 0.1479 & 0.52 & 1.22 & 0.4713 & 0.46 & 0.34 & 0.82 & 1.67 & 0.56 \\
\hline 31.5 & 0.14 & 0.29 & 0.11 & 0.0942 & 0.60 & 1.31 & 0.5506 & 0.50 & 0.39 & 0.70 & 1.46 & 0.32 \\
\hline 32.5 & 0.15 & 0.31 & 0.13 & 0.1060 & 0.55 & 1.19 & 0.5871 & 0.51 & 0.41 & 0.70 & 1.60 & 0.35 \\
\hline 33.5 & 0.15 & 0.33 & 0.13 & 0.1388 & 0.54 & 1.29 & 0.6118 & 0.45 & 0.41 & 0.79 & 1.55 & 0.42 \\
\hline 34.5 & 0.16 & 0.35 & 0.10 & 0.1264 & 0.57 & 1.44 & 0.6116 & 0.44 & 0.28 & 0.71 & 1.35 & 0.36 \\
\hline 35.5 & 0.23 & 0.46 & 0.13 & 0.1608 & 0.70 & 1.49 & 0.8146 & 0.49 & 0.29 & 0.67 & 1.24 & 0.35 \\
\hline 36.5 & 0.22 & 0.44 & 0.09 & 0.1100 & 0.49 & 0.90 & 0.7432 & 0.50 & 0.20 & 0.75 & 1.46 & 0.25 \\
\hline 37.5 & 0.21 & 0.39 & 0.09 & 0.0997 & 0.66 & 0.79 & 0.6890 & 0.53 & 0.23 & 0.70 & 1.51 & 0.25 \\
\hline 38.5 & 0.28 & 0.55 & 0.17 & 0.1922 & 0.82 & 1.28 & 1.0034 & 0.50 & 0.32 & 0.73 & 1.45 & 0.35 \\
\hline 39.5 & 0.25 & 0.33 & 0.16 & 0.2636 & 0.91 & 1.27 & 0.7434 & 0.77 & 0.49 & 0.70 & 0.00 & 0.80 \\
\hline 40.5 & 0.25 & 0.48 & 0.17 & 0.1434 & 0.71 & 1.20 & 0.8986 & 0.51 & 0.35 & 0.70 & 1.58 & 0.30 \\
\hline 41.5 & 0.24 & 0.47 & 0.18 & 0.1524 & 0.82 & 0.97 & 0.8947 & 0.50 & 0.39 & 0.70 & 1.59 & 0.32 \\
\hline 42.5 & 0.28 & 0.53 & 0.17 & 0.1501 & 0.87 & 0.95 & 0.9828 & 0.52 & 0.32 & 0.70 & 1.46 & 0.28 \\
\hline 43.5 & 0.27 & 0.55 & 0.23 & 0.1655 & 0.90 & 1.04 & 1.0439 & 0.49 & 0.42 & 0.80 & 1.62 & 0.30 \\
\hline 44.5 & 0.24 & 0.59 & 0.15 & 0.1357 & 0.52 & 0.83 & 0.9758 & 0.40 & 0.24 & 0.68 & 1.22 & 0.23 \\
\hline 45.5 & 0.31 & 0.57 & 0.21 & 0.1540 & 1.02 & 1.00 & 1.0923 & 0.53 & 0.37 & 0.72 & 1.33 & 0.27 \\
\hline 46.5 & 0.28 & 0.61 & 0.17 & 0.2329 & 0.86 & 1.17 & 1.0583 & 0.46 & 0.27 & 0.75 & 1.45 & 0.38 \\
\hline 47.5 & 0.28 & 0.60 & 0.20 & 0.1796 & 0.89 & 1.07 & 1.0789 & 0.46 & 0.34 & 0.79 & 1.54 & 0.30 \\
\hline 48.5 & 0.33 & 0.63 & 0.24 & 0.1880 & 1.03 & 1.11 & 1.1976 & 0.52 & 0.38 & 0.70 & 1.63 & 0.30 \\
\hline 49.5 & 0.26 & 0.53 & 0.16 & 0.1326 & 0.84 & 0.87 & 0.9443 & 0.49 & 0.31 & 0.69 & 1.36 & 0.25 \\
\hline 50.5 & 0.27 & 0.53 & 0.17 & 0.1713 & 0.81 & 0.91 & 0.9745 & 0.51 & 0.33 & 0.79 & 1.61 & 0.32 \\
\hline 51.5 & 0.33 & 0.68 & 0.20 & 0.1953 & 0.95 & 1.32 & 1.2145 & 0.49 & 0.30 & 0.76 & 1.34 & 0.29 \\
\hline 52.5 & 0.40 & 0.71 & 0.18 & 0.2140 & 0.94 & 1.05 & 1.2953 & 0.57 & 0.25 & 0.69 & 1.39 & 0.30 \\
\hline 53.5 & 0.29 & 0.60 & 0.24 & 0.1764 & 0.92 & 0.93 & 1.1337 & 0.49 & 0.40 & 0.82 & 1.56 & 0.29 \\
\hline 54.5 & 0.31 & 0.60 & 0.15 & 0.1932 & 0.97 & 1.01 & 1.0656 & 0.51 & 0.25 & 0.76 & 1.75 & 0.32 \\
\hline 55.5 & 0.53 & 0.99 & 0.32 & 0.2323 & 1.15 & 0.96 & 1.8391 & 0.53 & 0.33 & 0.67 & 1.34 & 0.24 \\
\hline 56.5 & 0.61 & 1.27 & 0.36 & 0.1990 & 0.72 & 0.88 & 2.2406 & 0.48 & 0.29 & 0.57 & 1.19 & 0.16 \\
\hline 57.5 & 1.10 & 2.16 & 0.93 & 0.2084 & 1.58 & 0.86 & 4.1854 & 0.51 & 0.43 & 0.58 & 1.03 & 0.10 \\
\hline 58.5 & 1.23 & 2.14 & 0.79 & 0.2092 & 1.69 & 0.87 & 4.1617 & 0.57 & 0.37 & 0.55 & 1.09 & 0.10 \\
\hline 59.5 & 1.21 & 2.33 & 0.93 & 0.1797 & 1.18 & 1.38 & 4.4684 & 0.52 & 0.40 & 0.57 & 1.07 & 0.08 \\
\hline
\end{tabular}




\begin{tabular}{|c|c|c|c|c|c|c|c|c|c|c|c|c|}
\hline 60.5 & 1.23 & 2.18 & 1.02 & 0.2039 & 1.73 & 1.04 & 4.4373 & 0.57 & 0.47 & 0.58 & 1.14 & 0.09 \\
\hline 61.5 & 1.35 & 2.77 & 0.79 & 0.2295 & 1.64 & 1.04 & 4.9075 & 0.49 & 0.28 & 0.61 & 1.05 & 0.08 \\
\hline 62.5 & 1.51 & 2.74 & 1.18 & 0.2587 & 1.90 & 1.27 & 5.4277 & 0.55 & 0.43 & 0.53 & 0.95 & 0.09 \\
\hline 63.5 & 1.80 & 3.20 & 1.22 & 0.2237 & 1.74 & 0.97 & 6.2276 & 0.56 & 0.38 & 0.47 & 0.85 & 0.07 \\
\hline 64.5 & 1.27 & 2.40 & 0.93 & 0.2790 & 1.81 & 1.26 & 4.6095 & 0.53 & 0.39 & 0.53 & 0.96 & 0.12 \\
\hline 65.5 & 1.15 & 2.07 & 0.94 & 0.2470 & 1.79 & 0.92 & 4.1586 & 0.55 & 0.45 & 0.56 & 1.07 & 0.12 \\
\hline 66.5 & 1.27 & 2.38 & 0.74 & 0.2618 & 1.79 & 1.02 & 4.3928 & 0.53 & 0.31 & 0.54 & 1.07 & 0.11 \\
\hline 67.5 & 1.00 & 1.79 & 0.85 & 0.1983 & 1.57 & 0.71 & 3.6389 & 0.56 & 0.48 & 0.61 & 1.21 & 0.11 \\
\hline 68.5 & 1.19 & 2.18 & 0.84 & 0.2506 & 1.76 & 0.91 & 4.1993 & 0.55 & 0.38 & 0.52 & 0.98 & 0.12 \\
\hline 69.5 & 0.85 & 1.71 & 0.53 & 0.1673 & 0.84 & 0.68 & 3.0966 & 0.50 & 0.31 & 0.61 & 1.18 & 0.10 \\
\hline 70.5 & 1.31 & 2.46 & 0.92 & 0.3261 & 1.78 & 0.97 & 4.7013 & 0.53 & 0.38 & 0.54 & 1.01 & 0.13 \\
\hline 71.5 & 1.25 & 2.32 & 1.02 & 0.2840 & 1.73 & 1.01 & 4.5828 & 0.54 & 0.44 & 0.58 & 1.09 & 0.12 \\
\hline 72.5 & 1.20 & 2.19 & 0.96 & 0.2213 & 1.81 & 0.93 & 4.3449 & 0.55 & 0.44 & 0.58 & 1.14 & 0.10 \\
\hline 73.5 & 1.04 & 2.07 & 0.72 & 0.2024 & 1.13 & 0.80 & 3.8276 & 0.50 & 0.35 & 0.55 & 1.03 & 0.10 \\
\hline 74.5 & 1.41 & 2.68 & 1.22 & 0.2547 & 1.97 & 1.01 & 5.3135 & 0.53 & 0.45 & 0.50 & 0.87 & 0.09 \\
\hline 75.5 & 1.39 & 2.62 & 1.16 & 0.3377 & 1.97 & 1.29 & 5.1729 & 0.53 & 0.44 & 0.61 & 1.08 & 0.13 \\
\hline 76.5 & 1.30 & 2.48 & 1.09 & 0.2550 & 1.93 & 1.23 & 4.8662 & 0.52 & 0.44 & 0.53 & 0.95 & 0.10 \\
\hline 77.5 & 1.14 & 2.00 & 1.07 & 0.2116 & 1.66 & 0.93 & 4.2050 & 0.57 & 0.53 & 0.54 & 1.06 & 0.11 \\
\hline 78.5 & 1.25 & 2.30 & 1.02 & 0.2549 & 1.81 & 1.06 & 4.5619 & 0.54 & 0.44 & 0.53 & 1.00 & 0.11 \\
\hline 79.5 & 1.23 & 2.15 & 1.02 & 0.2753 & 1.94 & 1.26 & 4.4096 & 0.57 & 0.48 & 0.55 & 1.17 & 0.13 \\
\hline 80.5 & 1.22 & 2.22 & 1.09 & 0.2613 & 1.99 & 1.02 & 4.5331 & 0.55 & 0.49 & 0.56 & 1.11 & 0.12 \\
\hline
\end{tabular}


Table S4. CuO-derived compounds (adapted from Tesi et al. 2014 and references therein). MS means that the compound has multiple sources but is relatively abundant in the source mentioned.

\begin{tabular}{|c|c|c|c|}
\hline Group name & Name & Abbreviation & Source \\
\hline \multirow{11}{*}{ 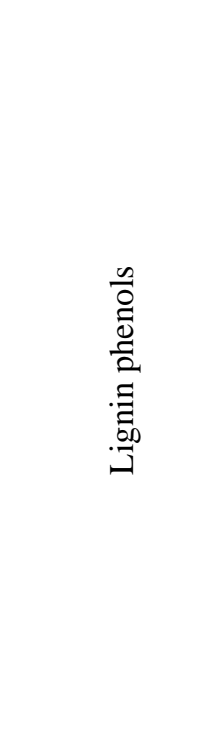 } & Vanillyl phenols & V & \multirow{3}{*}{$\begin{array}{l}\text { Cell walls of angiosperm/gymnosperm } \\
\text { vascular plants }\end{array}$} \\
\hline & Vanillin & $\mathrm{Vl}$ & \\
\hline & Acetovanillone & Vn & \\
\hline & Vanillic acid & Vd & \multirow{5}{*}{ Cell walls of angiosperm vascular plants } \\
\hline & Syringyl phenols & $S$ & \\
\hline & Syringaldehyde & $\mathrm{S} 1$ & \\
\hline & Acetosyringone & $\mathrm{Sn}$ & \\
\hline & Syringic acid & $\mathrm{Sd}$ & \\
\hline & Cinnamyl phenols & $\mathrm{C}$ & \multirow{3}{*}{ Non-woody vascular plant tissues } \\
\hline & $p$-Coumaric acid & $\mathrm{pCd}$ & \\
\hline & Ferulic acid & $\mathrm{Fd}$ & \\
\hline \multirow{5}{*}{ 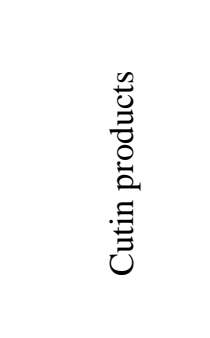 } & Hydroxyhexadecanoic acid & $\omega-\mathrm{C} 16$ & \multirow{5}{*}{$\begin{array}{l}\text { Leaves; blades and needles of vascular } \\
\text { plants }\end{array}$} \\
\hline & Hexadecan-1,16-dioic acid & C16DA & \\
\hline & 18-Hydroxyoctadec-9-enoic acid & $\omega-\mathrm{C} 18: 1$ & \\
\hline & 7 or 8-Dihydroxy C16 x, $\omega$-dioic acids & $\mathrm{x}-\mathrm{OH}, \mathrm{C} 16 \mathrm{DA}$ & \\
\hline & 8,9 or 10-Dihydroxy C16 acids & $\mathrm{x}, \omega-\mathrm{OH} \mathrm{C} 16$ & \\
\hline \multirow{3}{*}{ 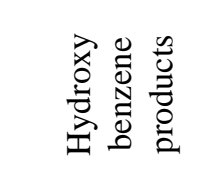 } & Benzoic acid & $\mathrm{Bd}$ & \multirow{2}{*}{$\begin{array}{l}\text { Phytoplankton } \\
\text { and soil (MS) }\end{array}$} \\
\hline & m-Hydroxybenzoic acid & $\mathrm{m}-\mathrm{Bd}$ & \\
\hline & 3,5-Dihydroxybenzoic acid & $3,5-\mathrm{Bd}$ & Abundant in soil \\
\hline \multirow{6}{*}{ 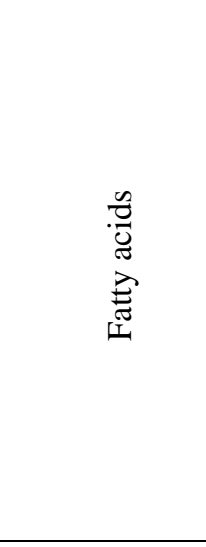 } & Octanoic acid & C8FA & Phytoplankton (MS) \\
\hline & Decanoic acid & C10FA & Bacteria \\
\hline & Dodecanoic acid & $\mathrm{C} 12 \mathrm{FA}$ & Bacteria (MS) \\
\hline & Tetradecanoic acid & C14FA & Phytoplankton (MS) \\
\hline & Hexadecanoic acids & C16FA:1, C16FA & $\begin{array}{l}\text { Phytoplankton and soil (also bacteria for } \\
\text { C16FA:1) (MS) }\end{array}$ \\
\hline & Octadecanoic acids & C18FA:1, C18FA & $\begin{array}{l}\text { Phytoplankton and soil (also bacteria for } \\
\text { C18FA:1) (MS) }\end{array}$ \\
\hline
\end{tabular}


Table S5. Source apportionment data from the Monte Carlo mixing model based on carbon isotopes $\left(\Delta^{14} \mathrm{C}, \delta^{13} \mathrm{C}\right)$ as fractions (mean and median, \%) for the sediment core GC58, including $5^{\text {th }}(\mathbf{q 0 5})$ and $9^{\text {th }}(\mathbf{q 9 5})$ percentiles and standard deviation (sd). Topsoil-PF (topsoil permafrost) refers to thaw of the active-layer permafrost, ICD-PF (Ice Complex deposit permafrost) represents old Pleistocene material from coastal erosion and marine OC (organic carbon) to primary production of phytoplankton.

\begin{tabular}{|c|c|c|c|c|c|c|c|c|c|c|c|c|c|c|c|}
\hline & ICD-P & & & & & Topsoil- & & & & & Marine $\mathrm{O}$ & & & & \\
\hline $\begin{array}{r}\text { Corrected } \\
\text { depth }(\mathrm{cm})\end{array}$ & mean & median & sd & q05 & q95 & mean & median & sd & q05 & q95 & mean & median & sd & q05 & q95 \\
\hline 3.5 & 0.407 & 0.406 & 0.050 & 0.328 & 0.489 & 0.230 & 0.210 & 0.120 & 0.066 & 0.450 & 0.363 & 0.377 & 0.094 & 0.196 & 0.498 \\
\hline 5.5 & 0.437 & 0.435 & 0.047 & 0.363 & 0.516 & 0.196 & 0.179 & 0.103 & 0.057 & 0.386 & 0.367 & 0.377 & 0.083 & 0.217 & 0.487 \\
\hline 6.5 & 0.451 & 0.449 & 0.046 & 0.379 & 0.529 & 0.181 & 0.165 & 0.095 & 0.053 & 0.358 & 0.368 & 0.378 & 0.079 & 0.225 & 0.481 \\
\hline 7.5 & 0.464 & 0.462 & 0.046 & 0.394 & 0.542 & 0.167 & 0.152 & 0.089 & 0.049 & 0.334 & 0.369 & 0.377 & 0.075 & 0.233 & 0.477 \\
\hline 9.5 & 0.499 & 0.497 & 0.045 & 0.431 & 0.578 & 0.133 & 0.120 & 0.074 & 0.038 & 0.273 & 0.368 & 0.374 & 0.065 & 0.251 & 0.462 \\
\hline 10.5 & 0.519 & 0.517 & 0.045 & 0.452 & 0.597 & 0.116 & 0.104 & 0.067 & 0.031 & 0.241 & 0.365 & 0.370 & 0.059 & 0.258 & 0.453 \\
\hline 11.5 & 0.536 & 0.534 & 0.045 & 0.470 & 0.615 & 0.101 & 0.090 & 0.061 & 0.025 & 0.216 & 0.362 & 0.366 & 0.055 & 0.264 & 0.444 \\
\hline 12.5 & 0.552 & 0.550 & 0.045 & 0.487 & 0.630 & 0.090 & 0.078 & 0.057 & 0.021 & 0.195 & 0.358 & 0.362 & 0.052 & 0.267 & 0.436 \\
\hline 13.5 & 0.597 & 0.595 & 0.044 & 0.534 & 0.674 & 0.060 & 0.050 & 0.045 & 0.010 & 0.142 & 0.342 & 0.346 & 0.045 & 0.265 & 0.412 \\
\hline 14.5 & 0.625 & 0.623 & 0.045 & 0.561 & 0.702 & 0.046 & 0.036 & 0.040 & 0.006 & 0.115 & 0.329 & 0.331 & 0.044 & 0.255 & 0.397 \\
\hline 15.5 & 0.642 & 0.641 & 0.046 & 0.575 & 0.719 & 0.038 & 0.029 & 0.037 & 0.004 & 0.101 & 0.319 & 0.321 & 0.044 & 0.246 & 0.388 \\
\hline 17.5 & 0.654 & 0.653 & 0.047 & 0.586 & 0.732 & 0.034 & 0.025 & 0.035 & 0.003 & 0.092 & 0.312 & 0.313 & 0.044 & 0.238 & 0.382 \\
\hline 18.5 & 0.653 & 0.652 & 0.047 & 0.587 & 0.731 & 0.035 & 0.025 & 0.035 & 0.003 & 0.093 & 0.312 & 0.314 & 0.044 & 0.238 & 0.381 \\
\hline 19.5 & 0.650 & 0.648 & 0.046 & 0.586 & 0.728 & 0.037 & 0.027 & 0.036 & 0.003 & 0.098 & 0.313 & 0.315 & 0.044 & 0.239 & 0.381 \\
\hline 20.5 & 0.645 & 0.643 & 0.046 & 0.583 & 0.724 & 0.040 & 0.030 & 0.037 & 0.004 & 0.105 & 0.314 & 0.317 & 0.044 & 0.239 & 0.382 \\
\hline 21.5 & 0.641 & 0.638 & 0.046 & 0.579 & 0.720 & 0.044 & 0.034 & 0.039 & 0.005 & 0.114 & 0.314 & 0.317 & 0.044 & 0.239 & 0.382 \\
\hline 22.5 & 0.639 & 0.635 & 0.045 & 0.578 & 0.718 & 0.049 & 0.039 & 0.041 & 0.005 & 0.123 & 0.312 & 0.315 & 0.044 & 0.236 & 0.380 \\
\hline 23.5 & 0.638 & 0.634 & 0.045 & 0.577 & 0.717 & 0.054 & 0.044 & 0.043 & 0.006 & 0.131 & 0.308 & 0.311 & 0.044 & 0.232 & 0.376 \\
\hline 24.5 & 0.639 & 0.635 & 0.045 & 0.578 & 0.719 & 0.059 & 0.049 & 0.044 & 0.008 & 0.140 & 0.302 & 0.305 & 0.044 & 0.226 & 0.370 \\
\hline 25.5 & 0.641 & 0.637 & 0.045 & 0.581 & 0.722 & 0.064 & 0.055 & 0.046 & 0.010 & 0.148 & 0.294 & 0.297 & 0.044 & 0.218 & 0.362 \\
\hline 26.5 & 0.646 & 0.641 & 0.045 & 0.585 & 0.727 & 0.070 & 0.061 & 0.047 & 0.011 & 0.156 & 0.285 & 0.287 & 0.044 & 0.209 & 0.353 \\
\hline
\end{tabular}




\begin{tabular}{|c|c|c|c|c|c|c|c|c|c|c|c|c|c|c|c|}
\hline 27.5 & 0.651 & 0.647 & 0.045 & 0.591 & 0.733 & 0.075 & 0.067 & 0.048 & 0.014 & 0.163 & 0.274 & 0.275 & 0.044 & 0.198 & 0.343 \\
\hline 28.5 & 0.658 & 0.654 & 0.045 & 0.598 & 0.740 & 0.081 & 0.073 & 0.049 & 0.016 & 0.170 & 0.261 & 0.263 & 0.044 & 0.185 & 0.331 \\
\hline 29.5 & 0.667 & 0.662 & 0.045 & 0.607 & 0.750 & 0.086 & 0.079 & 0.049 & 0.017 & 0.177 & 0.247 & 0.249 & 0.045 & 0.170 & 0.318 \\
\hline 30.5 & 0.676 & 0.670 & 0.045 & 0.618 & 0.760 & 0.091 & 0.084 & 0.050 & 0.019 & 0.183 & 0.233 & 0.235 & 0.045 & 0.155 & 0.304 \\
\hline 31.5 & 0.687 & 0.680 & 0.046 & 0.629 & 0.772 & 0.095 & 0.089 & 0.051 & 0.021 & 0.190 & 0.218 & 0.220 & 0.046 & 0.139 & 0.291 \\
\hline 32.5 & 0.699 & 0.691 & 0.046 & 0.642 & 0.786 & 0.099 & 0.093 & 0.052 & 0.021 & 0.196 & 0.202 & 0.205 & 0.047 & 0.122 & 0.277 \\
\hline 33.5 & 0.711 & 0.703 & 0.047 & 0.654 & 0.800 & 0.102 & 0.096 & 0.054 & 0.021 & 0.200 & 0.187 & 0.190 & 0.049 & 0.104 & 0.264 \\
\hline 34.5 & 0.724 & 0.716 & 0.047 & 0.668 & 0.817 & 0.103 & 0.097 & 0.055 & 0.020 & 0.205 & 0.172 & 0.175 & 0.050 & 0.087 & 0.251 \\
\hline 35.5 & 0.738 & 0.729 & 0.048 & 0.681 & 0.833 & 0.104 & 0.098 & 0.057 & 0.017 & 0.209 & 0.158 & 0.161 & 0.051 & 0.070 & 0.239 \\
\hline 36.5 & 0.753 & 0.743 & 0.049 & 0.695 & 0.849 & 0.103 & 0.097 & 0.059 & 0.015 & 0.211 & 0.144 & 0.147 & 0.052 & 0.055 & 0.227 \\
\hline 37.5 & 0.767 & 0.756 & 0.049 & 0.709 & 0.865 & 0.102 & 0.095 & 0.060 & 0.012 & 0.211 & 0.131 & 0.134 & 0.053 & 0.041 & 0.217 \\
\hline 38.5 & 0.781 & 0.770 & 0.050 & 0.722 & 0.879 & 0.099 & 0.092 & 0.061 & 0.009 & 0.213 & 0.120 & 0.121 & 0.054 & 0.030 & 0.207 \\
\hline 39.5 & 0.794 & 0.784 & 0.054 & 0.728 & 0.894 & 0.098 & 0.089 & 0.066 & 0.006 & 0.218 & 0.109 & 0.109 & 0.054 & 0.021 & 0.200 \\
\hline 40.5 & 0.606 & 0.633 & 0.141 & 0.328 & 0.789 & 0.141 & 0.091 & 0.146 & 0.011 & 0.448 & 0.253 & 0.253 & 0.076 & 0.132 & 0.376 \\
\hline 41.5 & 0.638 & 0.644 & 0.092 & 0.477 & 0.774 & 0.123 & 0.097 & 0.096 & 0.024 & 0.315 & 0.239 & 0.240 & 0.063 & 0.137 & 0.341 \\
\hline 42.5 & 0.654 & 0.652 & 0.066 & 0.552 & 0.762 & 0.116 & 0.102 & 0.068 & 0.032 & 0.248 & 0.230 & 0.231 & 0.057 & 0.137 & 0.322 \\
\hline 43.5 & 0.662 & 0.658 & 0.060 & 0.574 & 0.759 & 0.112 & 0.104 & 0.060 & 0.030 & 0.225 & 0.226 & 0.226 & 0.054 & 0.137 & 0.313 \\
\hline 44.5 & 0.666 & 0.664 & 0.061 & 0.576 & 0.766 & 0.108 & 0.101 & 0.059 & 0.025 & 0.218 & 0.225 & 0.225 & 0.053 & 0.139 & 0.312 \\
\hline 45.5 & 0.671 & 0.669 & 0.061 & 0.580 & 0.772 & 0.101 & 0.093 & 0.058 & 0.021 & 0.211 & 0.227 & 0.227 & 0.052 & 0.144 & 0.313 \\
\hline 46.5 & 0.679 & 0.676 & 0.060 & 0.590 & 0.778 & 0.092 & 0.083 & 0.055 & 0.017 & 0.196 & 0.229 & 0.229 & 0.051 & 0.149 & 0.313 \\
\hline 47.5 & 0.690 & 0.686 & 0.060 & 0.602 & 0.788 & 0.081 & 0.072 & 0.051 & 0.014 & 0.179 & 0.229 & 0.228 & 0.050 & 0.151 & 0.311 \\
\hline 48.5 & 0.699 & 0.695 & 0.060 & 0.610 & 0.797 & 0.075 & 0.066 & 0.049 & 0.012 & 0.169 & 0.227 & 0.226 & 0.049 & 0.150 & 0.308 \\
\hline 49.5 & 0.709 & 0.706 & 0.060 & 0.621 & 0.809 & 0.068 & 0.059 & 0.047 & 0.011 & 0.159 & 0.222 & 0.221 & 0.048 & 0.146 & 0.302 \\
\hline 50.5 & 0.722 & 0.719 & 0.061 & 0.633 & 0.824 & 0.062 & 0.053 & 0.045 & 0.009 & 0.150 & 0.215 & 0.214 & 0.048 & 0.140 & 0.294 \\
\hline 51.5 & 0.738 & 0.734 & 0.061 & 0.649 & 0.840 & 0.057 & 0.047 & 0.043 & 0.008 & 0.139 & 0.205 & 0.205 & 0.048 & 0.130 & 0.283 \\
\hline 52.5 & 0.753 & 0.749 & 0.062 & 0.664 & 0.855 & 0.052 & 0.042 & 0.041 & 0.006 & 0.132 & 0.195 & 0.194 & 0.047 & 0.121 & 0.272 \\
\hline 53.5 & 0.769 & 0.765 & 0.062 & 0.679 & 0.872 & 0.048 & 0.038 & 0.039 & 0.005 & 0.125 & 0.183 & 0.182 & 0.047 & 0.109 & 0.259 \\
\hline 54.5 & 0.786 & 0.783 & 0.063 & 0.696 & 0.887 & 0.044 & 0.034 & 0.038 & 0.004 & 0.118 & 0.170 & 0.169 & 0.047 & 0.096 & 0.246 \\
\hline 55.5 & 0.819 & 0.817 & 0.064 & 0.725 & 0.918 & 0.038 & 0.028 & 0.036 & 0.003 & 0.107 & 0.143 & 0.142 & 0.046 & 0.071 & 0.219 \\
\hline 56.5 & 0.848 & 0.850 & 0.065 & 0.749 & 0.944 & 0.034 & 0.023 & 0.037 & 0.002 & 0.104 & 0.118 & 0.116 & 0.045 & 0.048 & 0.194 \\
\hline 57.5 & 0.871 & 0.876 & 0.066 & 0.768 & 0.962 & 0.032 & 0.019 & 0.040 & 0.001 & 0.105 & 0.097 & 0.094 & 0.044 & 0.032 & 0.173 \\
\hline 58.5 & 0.888 & 0.896 & 0.067 & 0.782 & 0.976 & 0.031 & 0.017 & 0.042 & 0.001 & 0.105 & 0.081 & 0.076 & 0.042 & 0.020 & 0.155 \\
\hline
\end{tabular}




\begin{tabular}{|c|c|c|c|c|c|c|c|c|c|c|c|c|c|c|c|}
\hline 59.5 & 0.900 & 0.909 & 0.067 & 0.792 & 0.983 & 0.031 & 0.016 & 0.044 & 0.001 & 0.107 & 0.070 & 0.064 & 0.041 & 0.013 & 0.142 \\
\hline 60.5 & 0.906 & 0.915 & 0.066 & 0.798 & 0.988 & 0.032 & 0.017 & 0.044 & 0.000 & 0.111 & 0.063 & 0.057 & 0.040 & 0.009 & 0.133 \\
\hline 61.5 & 0.906 & 0.912 & 0.065 & 0.801 & 0.991 & 0.034 & 0.021 & 0.043 & 0.000 & 0.112 & 0.060 & 0.055 & 0.040 & 0.006 & 0.131 \\
\hline 62.5 & 0.897 & 0.899 & 0.064 & 0.797 & 0.992 & 0.041 & 0.030 & 0.042 & 0.000 & 0.119 & 0.062 & 0.057 & 0.042 & 0.004 & 0.136 \\
\hline 63.5 & 0.870 & 0.863 & 0.073 & 0.768 & 0.992 & 0.061 & 0.052 & 0.052 & 0.000 & 0.159 & 0.069 & 0.065 & 0.047 & 0.003 & 0.152 \\
\hline 64.5 & 0.718 & 0.832 & 0.281 & 0.075 & 0.981 & 0.180 & 0.072 & 0.235 & 0.002 & 0.738 & 0.102 & 0.076 & 0.092 & 0.010 & 0.294 \\
\hline 65.5 & 0.751 & 0.844 & 0.244 & 0.177 & 0.979 & 0.155 & 0.065 & 0.206 & 0.003 & 0.642 & 0.095 & 0.076 & 0.076 & 0.013 & 0.247 \\
\hline 66.5 & 0.765 & 0.842 & 0.222 & 0.253 & 0.980 & 0.142 & 0.065 & 0.188 & 0.002 & 0.575 & 0.093 & 0.078 & 0.071 & 0.012 & 0.230 \\
\hline 67.5 & 0.778 & 0.840 & 0.200 & 0.336 & 0.982 & 0.129 & 0.063 & 0.167 & 0.002 & 0.500 & 0.093 & 0.080 & 0.067 & 0.010 & 0.219 \\
\hline 68.5 & 0.794 & 0.841 & 0.175 & 0.427 & 0.983 & 0.113 & 0.061 & 0.144 & 0.002 & 0.415 & 0.093 & 0.082 & 0.063 & 0.010 & 0.207 \\
\hline 69.5 & 0.811 & 0.843 & 0.147 & 0.518 & 0.982 & 0.097 & 0.057 & 0.119 & 0.002 & 0.334 & 0.092 & 0.084 & 0.059 & 0.010 & 0.194 \\
\hline 70.5 & 0.827 & 0.845 & 0.120 & 0.608 & 0.980 & 0.083 & 0.054 & 0.093 & 0.002 & 0.260 & 0.090 & 0.085 & 0.054 & 0.011 & 0.184 \\
\hline 71.5 & 0.840 & 0.846 & 0.097 & 0.679 & 0.980 & 0.073 & 0.054 & 0.071 & 0.002 & 0.201 & 0.087 & 0.083 & 0.050 & 0.011 & 0.173 \\
\hline 72.5 & 0.851 & 0.847 & 0.080 & 0.730 & 0.980 & 0.066 & 0.055 & 0.055 & 0.002 & 0.168 & 0.083 & 0.081 & 0.047 & 0.011 & 0.163 \\
\hline 73.5 & 0.856 & 0.848 & 0.074 & 0.756 & 0.982 & 0.064 & 0.055 & 0.050 & 0.002 & 0.159 & 0.079 & 0.077 & 0.045 & 0.010 & 0.156 \\
\hline 74.5 & 0.857 & 0.854 & 0.081 & 0.730 & 0.984 & 0.068 & 0.056 & 0.058 & 0.002 & 0.176 & 0.076 & 0.072 & 0.045 & 0.009 & 0.153 \\
\hline 75.5 & 0.851 & 0.862 & 0.101 & 0.673 & 0.986 & 0.076 & 0.055 & 0.078 & 0.002 & 0.221 & 0.073 & 0.067 & 0.047 & 0.007 & 0.155 \\
\hline 76.5 & 0.840 & 0.867 & 0.128 & 0.590 & 0.988 & 0.088 & 0.055 & 0.103 & 0.001 & 0.283 & 0.071 & 0.064 & 0.050 & 0.006 & 0.161 \\
\hline 77.5 & 0.826 & 0.869 & 0.158 & 0.501 & 0.990 & 0.102 & 0.056 & 0.130 & 0.001 & 0.361 & 0.072 & 0.061 & 0.055 & 0.005 & 0.173 \\
\hline 78.5 & 0.809 & 0.869 & 0.187 & 0.402 & 0.992 & 0.117 & 0.057 & 0.154 & 0.001 & 0.442 & 0.074 & 0.060 & 0.061 & 0.005 & 0.189 \\
\hline 79.5 & 0.779 & 0.861 & 0.229 & 0.253 & 0.992 & 0.139 & 0.059 & 0.187 & 0.001 & 0.561 & 0.083 & 0.064 & 0.074 & 0.004 & 0.229 \\
\hline 80.5 & 0.724 & 0.836 & 0.282 & 0.090 & 0.995 & 0.171 & 0.067 & 0.225 & 0.000 & 0.687 & 0.105 & 0.079 & 0.101 & 0.002 & 0.313 \\
\hline
\end{tabular}

* Corrected depth is the original depth $+3 \mathrm{~cm}$ to account for core top loss during sampling (Sect 2.4). 


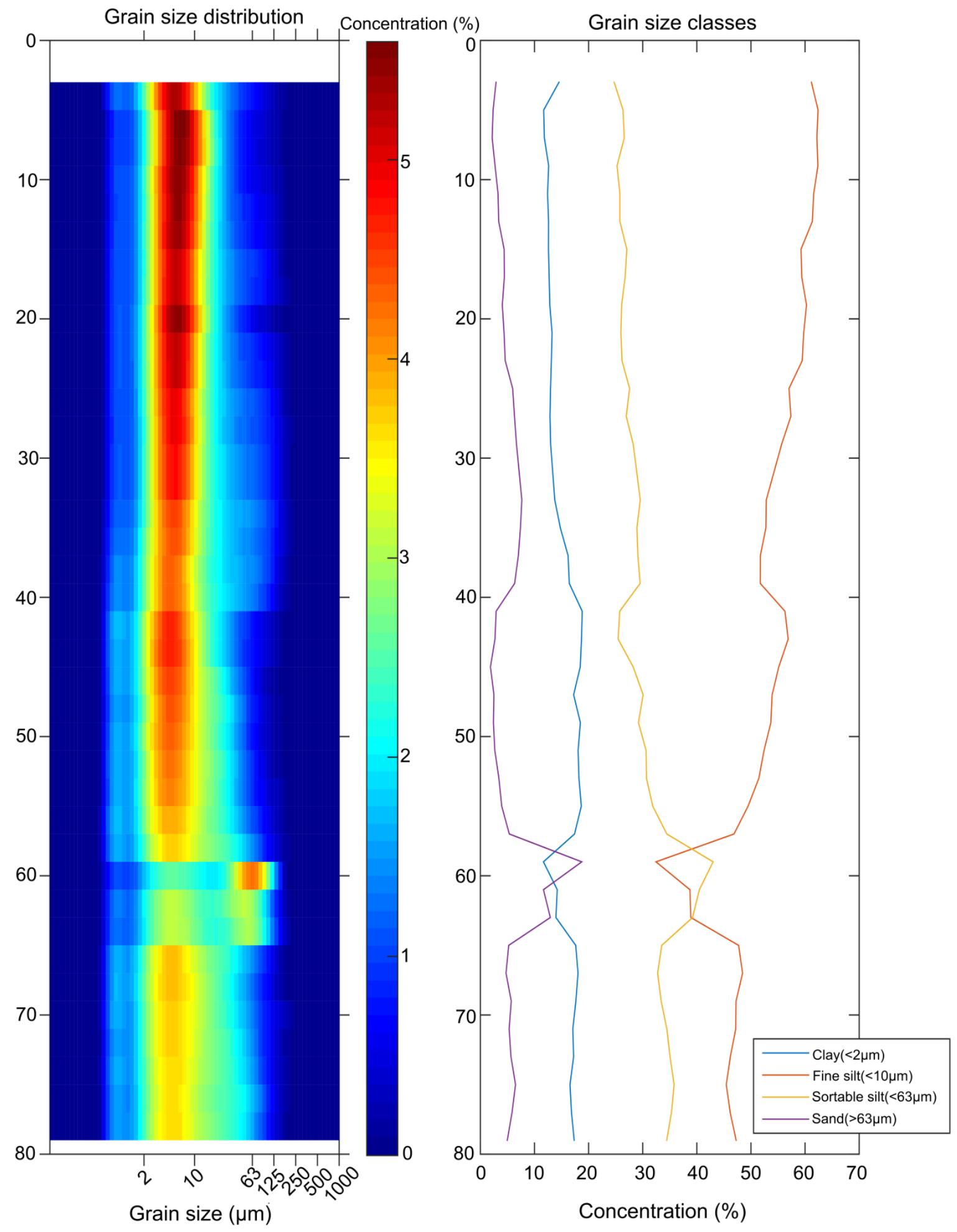

Figure S2. The grain size of the sediment core GC58. The GC58 core consists mainly of clay and silt with a fraction of sand around $60 \mathrm{~cm}$ of depth. 


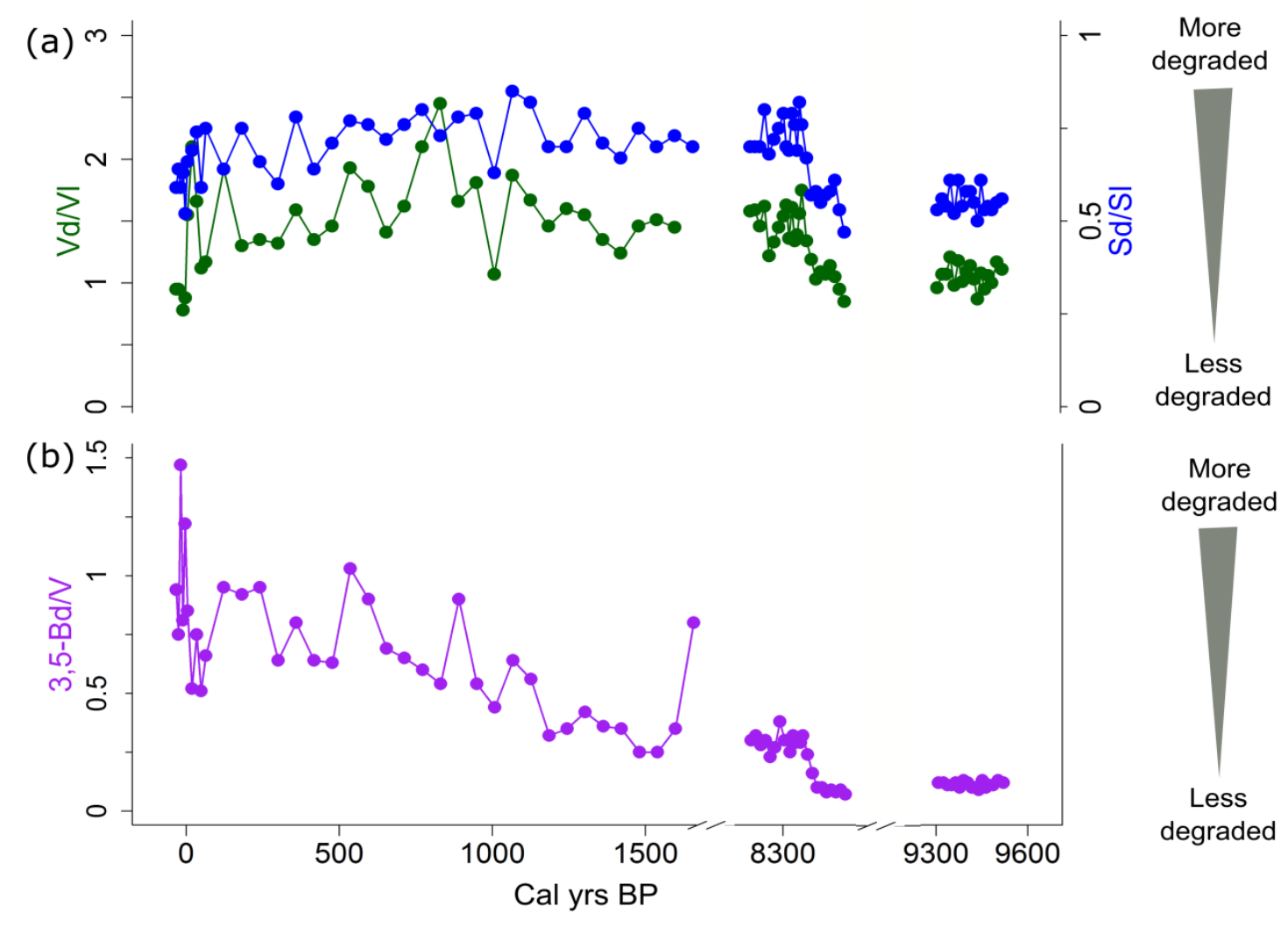

Figure S3. Degradation proxies for terrestrial organic carbon in the sediment core GC58. The $x$-axis has breaks due to gaps in the sediment chronology. (a) Syringyl acid to syringaldehyde ( $\mathbf{S d} / \mathbf{S I}$ ) and vanillic acid to vanillin $(\mathrm{Vd} / \mathrm{Vl})$ ratios are a lignin-phenol based degradation proxy. The variability at the core top may also reflect the analytical uncertainty caused by very low lignin concentrations. (b) Also the ratio of 3,5dihydrobenzoic acid to vanillyl phenols $(3,5-\mathrm{Bd} / \mathrm{V})$ provides information on degradation of terrestrial organic carbon. Higher values imply more degraded material for all the ratios as illustrated with the grey triangles. The 3,5-Bd/V values suggest a gradual increase in degradation from the bottom of the core to the top. 


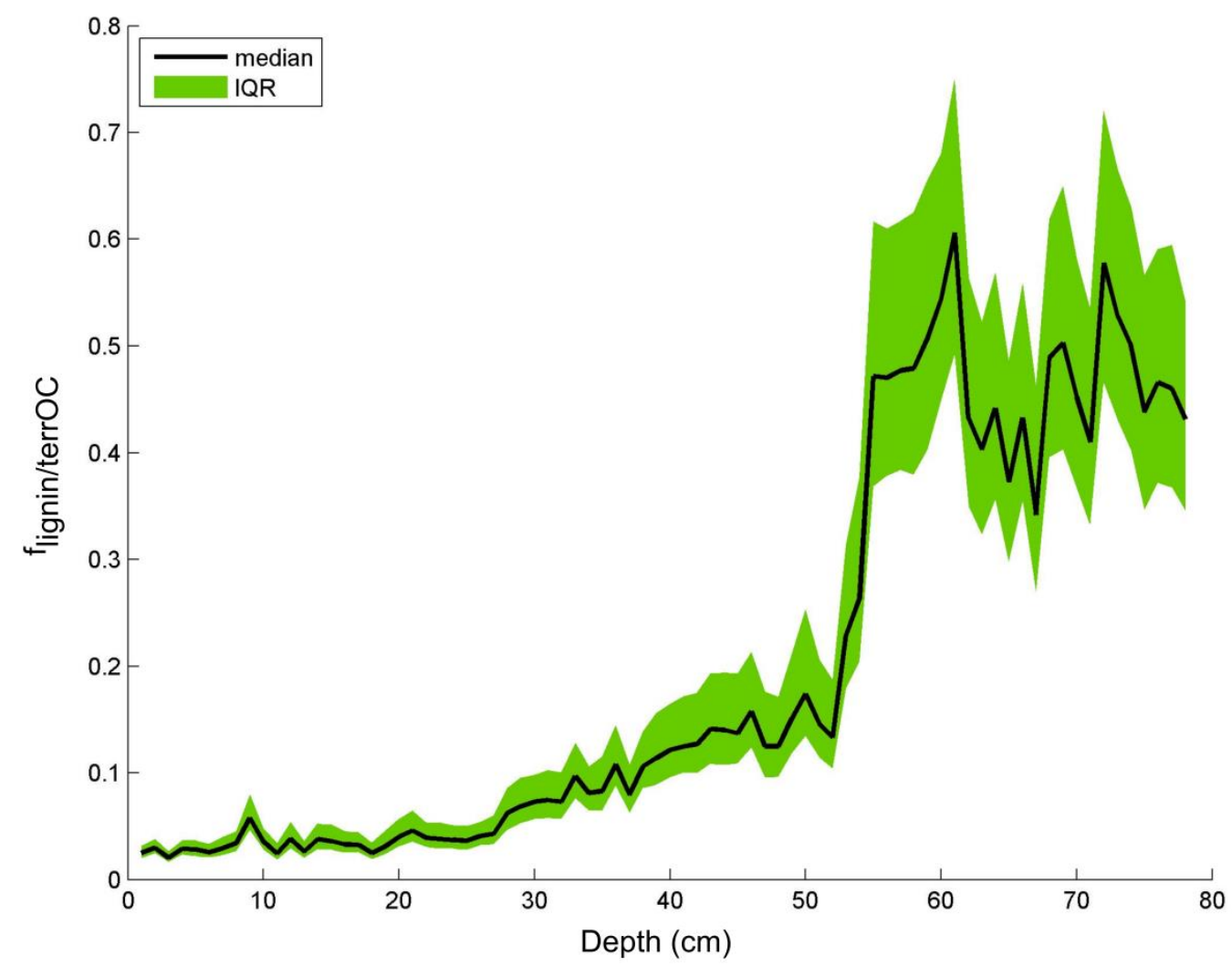

Figure S4. The ratio between the observed and expected lignin and terrestrial organic carbon (terrOC) ratios $\left(f_{\text {lig/terroc }}\right)$ with the interquartile range (IQR) for the sediment core GC58. The expected lignin values i.e. nondegraded lignin are taken from Tesi et al., (2016). See Supplementary methods for details. 


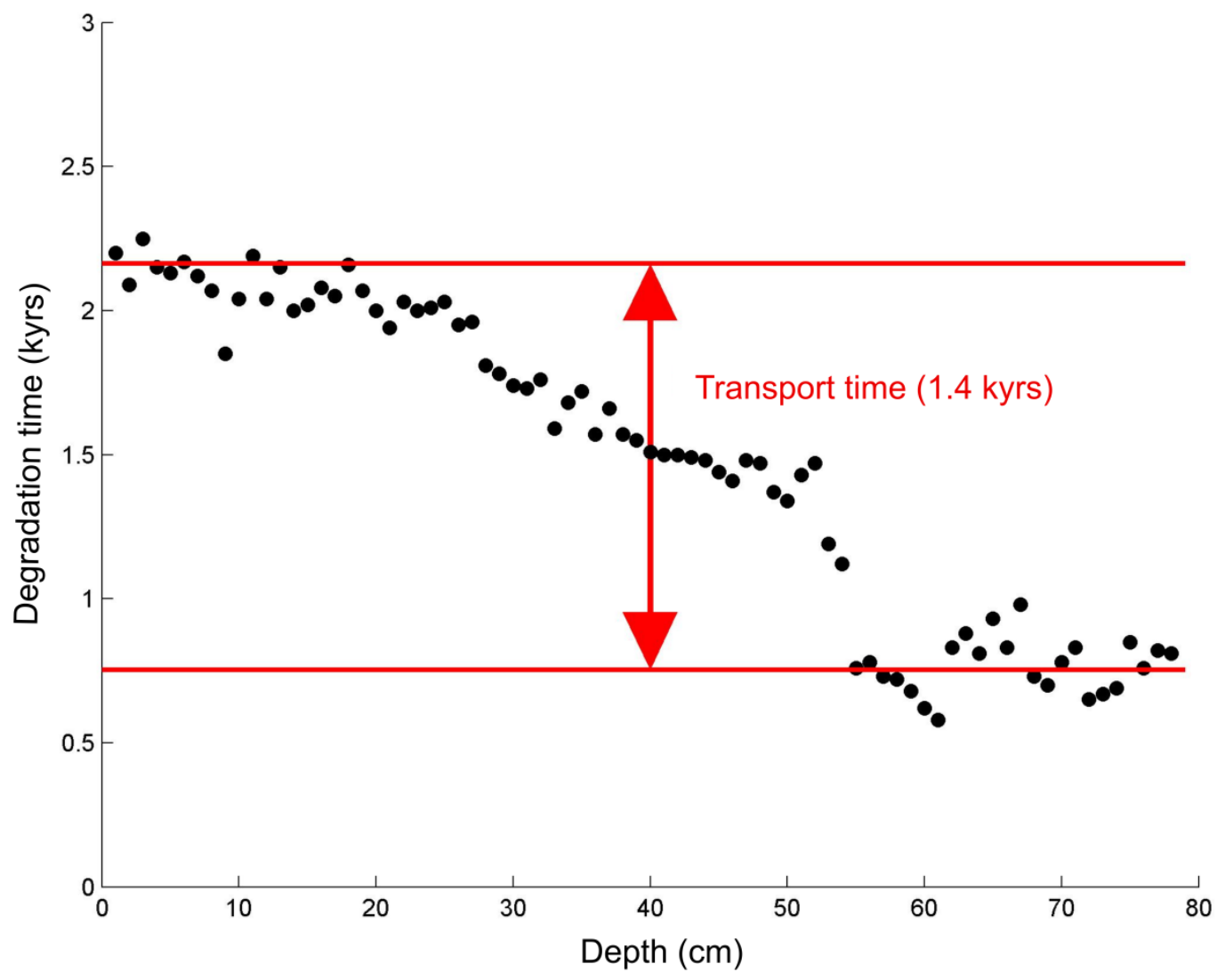

Figure S5. An estimate of the lateral transport time of sediments shown as the degradation time (kyr) against the core depth $(\mathrm{cm})$ in the sediment core GC58. See Supplementary methods for lateral transport time calculations. 


\section{Supplementary methods}

\section{Source apportionment calculations}

In the model for smoothly varying source proportions, data is split into three time-segments of contiguous observations. For each segment, observed $\delta^{13} C$ and $\Delta^{14} C$ are modelled as

$$
\begin{aligned}
& \delta^{13} C_{i}=\delta^{13} C^{I C D-P F} \times p_{i}^{I C D-P F}+\delta^{13} C^{T S-P F} \times p_{i}^{T S-P F}+\delta^{13} C^{M O C} \times p_{i}^{M O C}+\epsilon_{i}^{13}, \\
& \Delta^{14} C_{i}=\Delta^{14} C^{I C D-P F} \times p_{i}^{I C D-P F}+\Delta^{14} C^{T S-P F} \times p_{i}^{T S-P F}+\Delta^{14} C^{M O C} \times p_{i}^{M O C}+\epsilon_{i}^{14},
\end{aligned}
$$

where $\epsilon_{i}^{13}$ and $\epsilon_{i}^{14}$ are independent zero-mean normally distributed residuals with variances $\sigma_{13}^{2}$ and $\sigma_{14}^{2}$ respectively. Residual variances are assumed equal for the three time-segments. ICD-PF refers to Ice Complex deposit permafrost, TS-PF to topsoil permafrost and M OC to marine organic carbon.

The endmember values $\delta^{13} C^{I C D-P F}, \delta^{13} C^{T S-P F}, \delta^{13} C^{M O C}, \delta^{14} C^{I C D-P F}, \delta^{14} C^{T S-P F}$ and $\delta^{14} C^{M O C}$ are assumed random effects shared within each time-segment and independent between. With the exception of $\delta^{14} C^{I C D-P F}$ they are assumed normally distributed, with means and standard deviations reported earlier. To avoid values below $-1000, \delta^{14} C^{I C D-P F}+1000$ is instead assumed to be exponentially distributed. Due to different times of deposit, $\delta^{14} C^{I C D-P F}$ means are set to $-933,-833$ and -800 for the younger, middle and older segment respectively.

In order to account for the time-dependence between proportions, we follow an approach related to that of Parnell et al. (2013) by modelling $p_{i}^{I C D-P F}, p_{i}^{T S-P F}$ and $p_{i}^{M} O C$ using Bayesian cubic B-splines after a transformation to the real plane using the additive log-ratio transform,

$$
\begin{aligned}
& p_{i}^{I C D-P F}=\frac{\exp \left(s_{1}\left(y_{i}\right)\right)}{\exp \left(s_{1}\left(y_{i}\right)\right)+\exp \left(s_{2}\left(y_{i}\right)\right)+1^{\prime}} \\
& p_{i}^{T S-P F}=\frac{\exp \left(s_{2}\left(y_{i}\right)\right)}{\exp \left(s_{1}\left(y_{i}\right)\right)+\exp \left(s_{2}\left(y_{i}\right)\right)+1}
\end{aligned}
$$

and $p_{i}^{M O C}=1-p_{i}^{I C D-P F}-p_{i}^{T S-P F}$. The functions $s_{1}$ and $s_{2}$ are the spline-functions and $y_{i}$ estimated years BP for observation $i$. Separate/independent splines are used for each of the three time-segments and knots are placed at the centres and endpoints. The model is fitted using rjags (Plummer, 2016) within the R computing environment ( $\mathrm{R}$ Core Team, 2016). The code for the model is available at https://github.com/mskoldSU/Keskitalo_et_al.

\section{Lateral transport time estimation}

The GC58 core spans over a time period of $\sim 9,500$ cal yrs BP, during which the study area experienced a significant sea level rise (34 $\mathrm{m}$ in water depth). This means that the time for lateral transport of the terrestrial organic carbon (terrOC) from the shore to the site of sedimentation increased. To model this transport time the ratio of lignin/terrOC was used as a molecular clock. The fraction remaining lignin/terrOC $\left(\mathrm{f}_{\text {lig/terrOC }}\right)$ from remineralisation depends on the degradation of both lignin and terrOC. Bröder et al. (2015) established the following relation for the Laptev Sea (time $t$ in kyrs):

$$
\mathrm{f}_{\text {lig/terroc }}(\mathrm{t})=\frac{\mathrm{e}^{-2.6 \cdot \mathrm{t}}}{0.87 \cdot \mathrm{e}^{-2.2 \cdot \mathrm{t}}+0.13}
$$


The observational concentration terrOC can be established as $\mathrm{OC} /\left(1-\mathrm{f}_{\text {marine }}\right)$, where the fraction marine is derived from the source apportionment results. To obtain the observational $f_{\text {lig/terrOC }}$ we need to consider the expected lignin/terrOC signal for the sources that have not been degraded. For ICD-PF the lignin/terrOC ratio is $17.4 \pm 8.3 \mathrm{mg}$ $\mathrm{g}^{-1}$ and for topsoil-PF $20.9 \pm 6.4 \mathrm{mg} \mathrm{g}^{-1}$ (Tesi et al., 2016). Since the relative proportions of ICD-PF and topsoil-PF for each data point is known from the source apportionment, the non-degraded lignin/terrOC signatures may be estimated, using Markov chain Monte Carlo (MCMC) techniques to account for the endmember variability. By computing the ratio of the observed and non-degraded lignin/terrOC ratios we can calculate the fraction remaining lignin/terrOC and further, estimate the lateral transport times using Eq. (2).

\section{References}

Appleby, P. G. and Oldfield, F.: The calculation of lead-210 dates assuming a constant rate of supply of unsupported ${ }^{210} \mathrm{~Pb}$ to the sediment, Catena, 5, 1-8, 1978.

Bröder L., Tesi T., Semiletov I.P., and Gustafsson Ö.: Fate of permafrost-released organic matter in the Laptev Sea: What is its lateral transport time along the transect from the Lena delta area to the deep sea of the Arctic interior?, AGU Fall Meeting, San Francisco, USA, 14-18 December 2015, C33F-06, 2015.

Parnell, A. C., Phillips, D. L., Bearhop, S., Semmens, B. X., Ward, E. J., Moore, J. W., Jackson, A. L. Kelly, D. J and Inger, R.: Bayesian Stable Isotope Mixing Models, , Environmetrics, 24(6), 387-399, doi:10.1002/env.2221, 2013.

Plummer, M.: rjags: Bayesian Graphical Models using MCMC., 2016.

R Core Team: R: A language and environment for statistical computing, 2016.

Tesi, T., Semiletov, I., Hugelius, G., Dudarev, O., Kuhry, P. and Gustafsson, Ö.: Composition and fate of terrigenous organic matter along the Arctic land-ocean continuum in East Siberia: Insights from biomarkers and carbon isotopes, Geochim. Cosmochim. Acta, 133, 235-256, doi:10.1016/j.gca.2014.02.045, 2014.

Tesi, T., Muschitiello, F., Smittenberg, R. H., Jakobsson, M., Vonk, J. E., Hill, P., Andersson, A., Kirchner, N., Noormets, R., Dudarev, O., Semiletov, I. and Gustafsson, Ö.: Massive remobilization of permafrost carbon during post-glacial warming, Nat. Commun., 7, 13653, doi:10.1038/ncomms13653, 2016. 